\title{
Application of ESP32 as a Media for Learning Ozone Damage in the Form of IoT-Based Ultraviolet Index Readers
}

\author{
Nugroho Adi Pramono a, *, Fauzan Ibnu Sofyan ${ }^{\text {b }}$, Bertha Anggita Purwandani ${ }^{\text {c, Oktavia }}$ \\ Ghaisyani $^{\mathrm{d}}$ \\ ${ }^{a, b, c, d}$ Department of Physics, Universitas Negeri Malang \\ Malang, East Java, Indonesia
}

*Corresponding author's e-mail: nugroho.adi.fmipa@um.ac.id

\begin{abstract}
Ultraviolet light is part of the electromagnetic waves from solar radiation. Excessive UV light can cause damage to human skin. High UV index caused by one of the damage to the ozone layer, therefore it is important we study the ozone damage in an area. On this occasion made learning media in the form of an IoT-based ultraviolet index reader. This tool uses ESP32 as a controller by adding several modules namely UV index sensor and wifi module. The existence of a wifi module makes it easy for us to send data and control ESP32 using the internet, therefore this tool is said to be based on the Internet of Things (IoT). With this tool we can compare the UV Index level in several regions simultaneously without being in that area. From the difference in index, we can study the level of UV radiation in each region in real time. Therefore we can study the comparison of ozone damage levels.
\end{abstract}

\section{Introduction}

In everyday life, we really need sunlight for our survival. Plants really need sunlight for photosynthesis, humans need sunlight for vitamin D intake. Ultraviolet radiation is part of the electromagnetic waves from solar radiation. Excessive UV rays can cause damage to human skin. One of the causes of high UV index is the damage to the ozone layer, therefore it is important for us to study ozone damage. We can study the extent of ozone damage by comparing the UV index in several regions simultaneously.

The Ultraviolet light that is harmful to living things on earth is caused by many factors, one of which is global warming. Basically, global warming is one of the phenomena of increasing the average temperature of the atmosphere globally, either at sea or on Earth's land. Meanwhile, 
according to researchers from CIFOR (Center for International Forestry Research) global warming is an event of trapping of long-wave solar radiation emitted to the earth by greenhouse gases. Greenhouse gases have six types, namely Carbon Dioxide (CO), Methane (CH4), Nitrous Oxide (N2O) Hydroperfluorocarbons (HFCs), Perfluorocarbons (CFCs), and Sulfur Hexafloride (SF6), the six gases are naturally present in the atmosphere. The trapping of the sun's heat in the earth's atmosphere is called the Greenhouse Effect. In addition, the depletion of the ozone layer can also heat up the earth's temperature. Because, the thinner top layers of the atmosphere will make ultraviolet rays more freely emit radiation entering the earth, so that more and more ultraviolet rays will pass through the earth's surface and light cannot be reflected on the earth's surface. The less ultraviolet light on the surface of the earth, the light will be reflected easily by the Earth, while the more ultraviolet rays on the surface of the earth, the light will be difficult to be reflected [4].

In studying the level of ozone damage is constrained by comparing the UV index in real time and simultaneously in several areas. Based on these problems, research was carried out on the development of a UV index reader system based on the Internet of Things. Internet of Things is defined as the interconnection of embedded computing devices that are uniquely identified in the existence of the internet infrastructure. Internet of Things is a computing concept that describes a future where every physical object can be connected to the internet and can identify by itself between other devices [2].

The development of this tool aims to facilitate learning about ozone damage in an area by realtime and simultaneous data collection. This tool is designed to use the Esp 32 module as a processor and the GUVA-S12SD analog UV sensor module to read the UV index.

\section{Theory Basis}

\section{a. Remote Control System}

Remote control system which is used to control electronics is actually an example of a system control. Remote control system for setting electronic equipment generally uses a button press as controller input. In the control system long distance, broadly speaking there are two the main components, namely the local controller and remote control section. Local controllers are the control part by the operator, which is the part where controller gives access control, meanwhile the remote control part is the yang part directly related to that equipment controlled [1].

\section{b. Internet of Things}

Internet of Things (IoT) is the latest communication paradigm envisioning a near future, where objects from everyday life will be equipped with microcontrollers, wave transmitters for digital communication, and a suitable protocol stack will make them. able to communicate with each other and with users, so that it becomes an inseparable part of the internet 


\section{c. ESP 32}

Esp32 is an electronic circuit board in which there is a microcontroller where there is already a wifi module on the chip, this supports this module to create an internet of things application system. Esp 32 has several pin outs that function as input and output. The pin out consists of analog digital converter, digital analog converter, pulse width modulation, UART, I2C, I2S, SPI.

\section{d. Ultraviolet Light}

Ultraviolet (UV) light is a from of electromagnetic radiation with wavelengt from $10 \mathrm{~nm}$ t0 400nm, shorter than visible light but longer than X-rays[3]. Solar radiation that reaches the earth's surface has a wavelength of about $100 \mathrm{~nm}$ to $1 \mathrm{~mm}$. Ultraviolet light is divided into three parts, namely UV A, UV B, and UV C, with the following wavelength details

1. UV A $=315 \mathrm{~nm}-400 \mathrm{~nm}$

2. UV B $=280 \mathrm{~nm}-325 \mathrm{~nm}$

3. UV C $=100 \mathrm{~nm}-280 \mathrm{~nm}$

Based on the difference in wavelengths of the three types of UV rays, generally when entering the atmosphere, almost all UV C rays are retained in the ozone layer and $90 \%$ of UV B will be absorbed by the ozone layer, water vapour, and other gases in the atmosphere. Meanwhile, UV A rays will reach the earth's surface. Thus, the total ultraviolet rays contained in solar radiation when it reaches the earth's surface are UV A rays with a percentage of 90-99\% and a small percentage of UV B rays with a percentage of $<10 \%$. Therefore, the level of exposure to ultraviolet radiation on the earth's surface has both benefits and dangers for the survival of living things on earth. The level of exposure is described using a unit number called the UV index. The UV index is useful for monitoring the level of beneficial or harmful ultraviolet rays. The UV index scale for moderate hazard is at wavelengths between 250nm and 400nm [5].

\section{e. Sensor GUVA-S12SD}

The GUVA-S12SD uv sensor module is an analog sensor module used to detect UV radiation intensity. This sensor is capable of detecting UV rays with a wavelength of $200-370 \mathrm{~nm}$, so this sensor is capable enough to measure the intensity of UV rays. The module provides a standard, right-angled, 3-pin male-header interface connection:

1. GND: 0V (Ground connection)

2. VCC: $3.3 \mathrm{~V}$ to $5.5 \mathrm{~V}$ (5mA current consumption)

3. OUT: $0 \mathrm{~V}$ to $1 \mathrm{~V}$ ( 0 to $10 \mathrm{UV}$ Index)

Due to the $0 \mathrm{~V}$ to $1 \mathrm{~V}$ output range the UV index $=\mathrm{Vo} / 0.1 \mathrm{~V}$ 


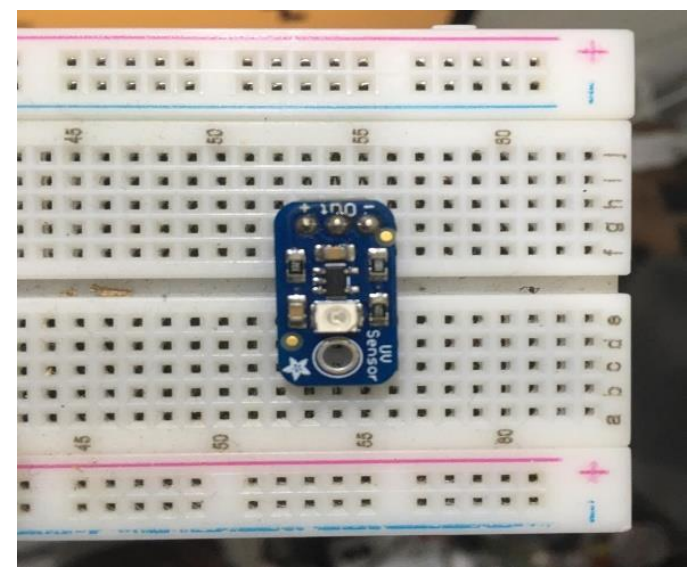

Figure 1. UV sensor type GUVA-S12SD

\section{Research Method}

The design of this IoT requires several materials, namely the ESP-32 module as a controller, the GUVA-S12SD UV sensor module as a UV index analog sensor, smartphone / PC as a tool to monitor data sent by the controller, power supply / power bank, and the internet network.

The system design in the form of a flowchart can be seen in the following figure 2 :

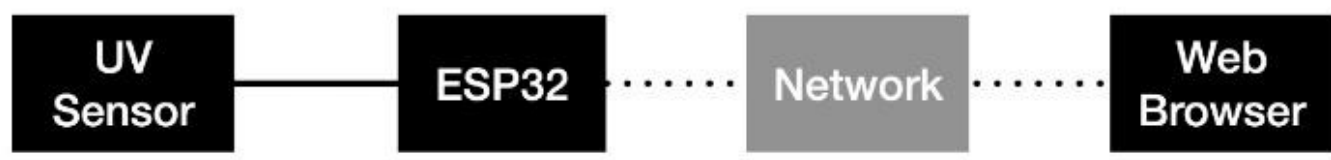

Figure 2. Design chart of an ultraviolet detection system

The hardware configuration can be seen in the following figure 3:

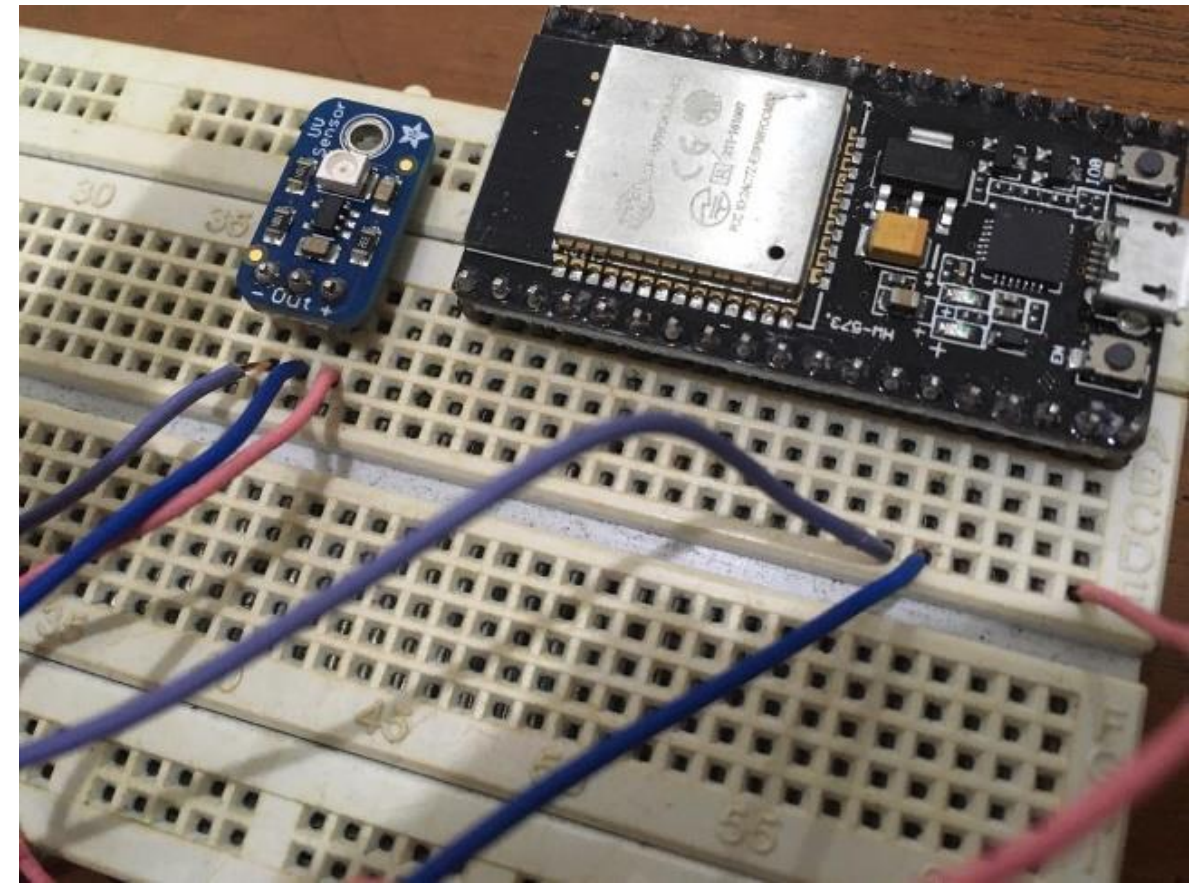

Figure 3. Hardware configuration 
The UV sensor (GUVA-S12SD) is given a voltage coming from the controller board and the output from the sensor is connected to the analog digital converter pins on the controller board. To provide voltage to the controller board we use Powerbank for practicality problems.

After the hardware configuration is complete, continuethe command of proggraming that the system does when turned on. Programming can be divided into two parts, namely: the first part UV sensor readings and the second part sending data obtained through internet connectivity

In programming the sensor reading, we use the else if method. This method divides the minimum output range to the maximum output into 11 parts so that the data obtained can be converted into $11 \mathrm{UV}$ indices. While programming data sending to servers via the internet, we program data transmission at a specified time interval.

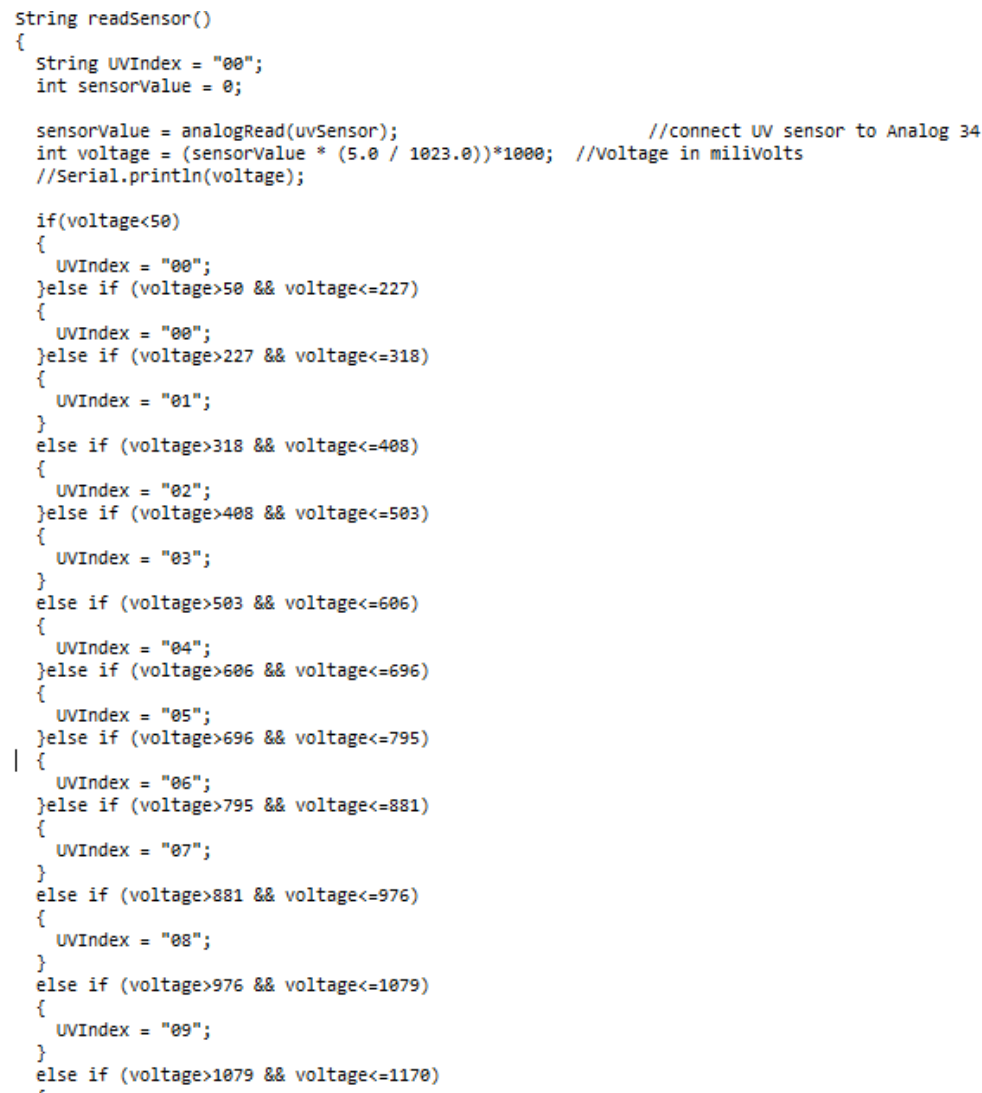

Figure 4. Code UV sensor reading 

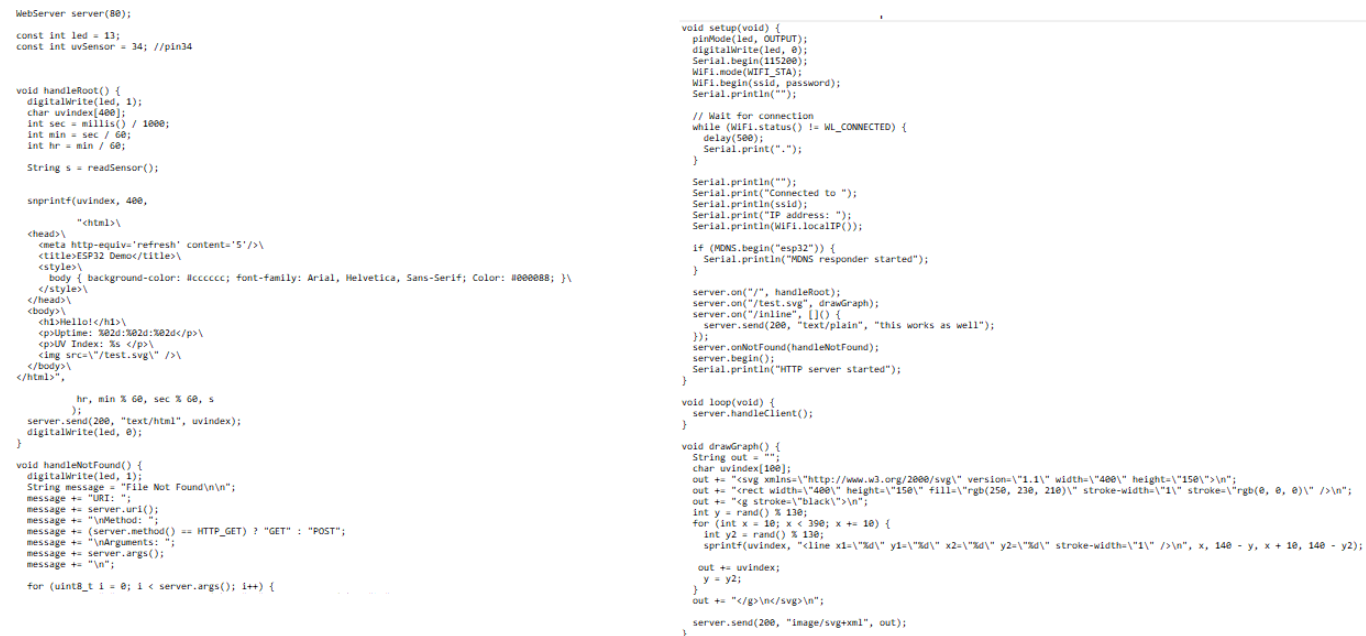

Figure 5. Code sending data to server

\section{Results and Discussion}

The first test was conducted to determine the performance of the application of the internet of things. This first test checks whether the program is running properly and also checks the connectivity to the server via the internet. To test the application of the internet of things, a laptop / smartphone is needed as a data monitoring tool.

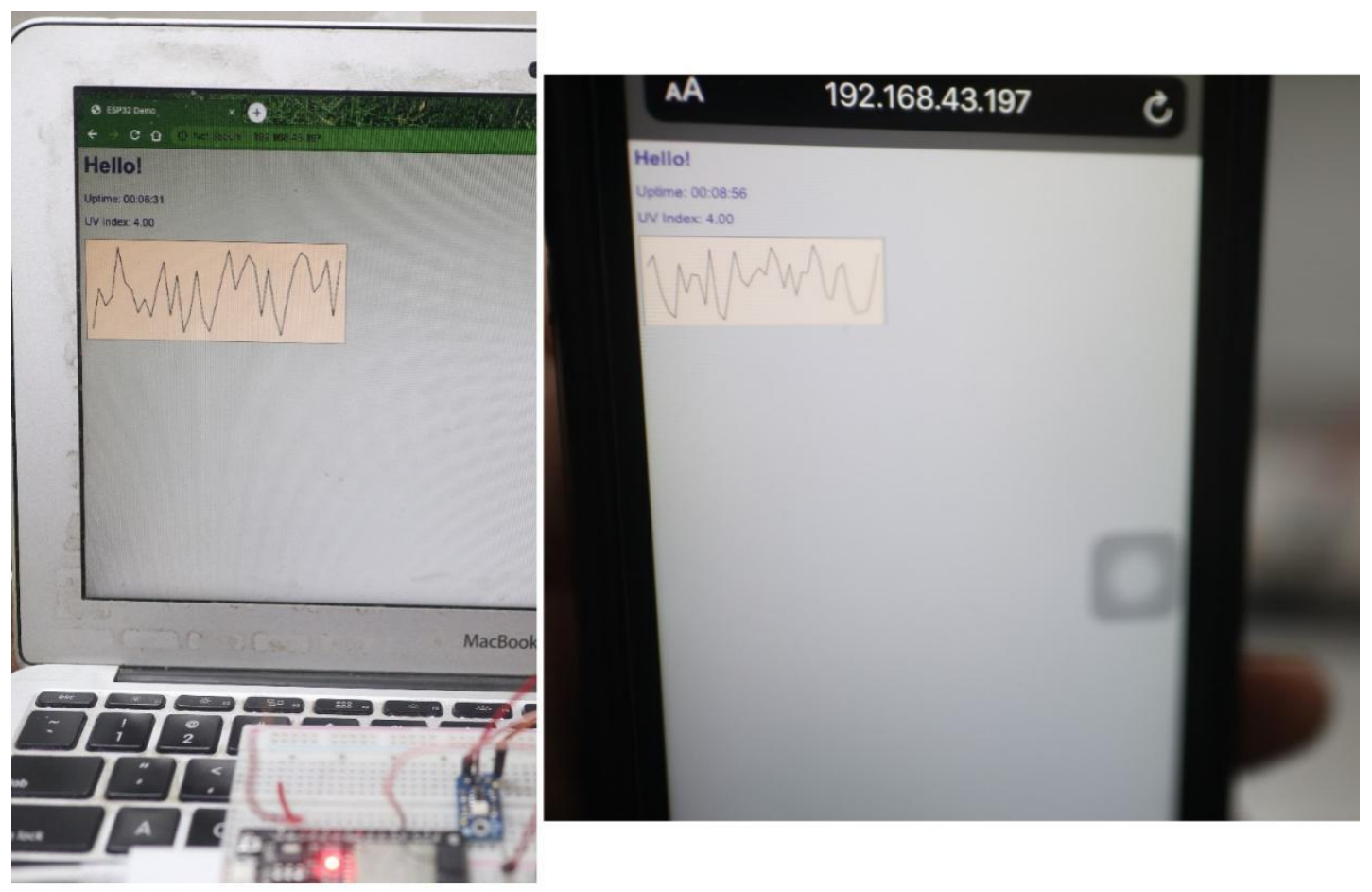

Figure 6. Testing application of the internet of thing

This test obtained satisfactory results. The program ran smoothly and the application of internet of things was successfully implemented.

The final test was carried out in two different places at the same time. The aim is to test the use of esp32 as a learning medium in the form of an IoT-based UV index reader. UV index results obtained from testing at two different locations as in the table below. 
Tabel 1.

Index Data Results Obtained

\begin{tabular}{lccc}
\hline \multicolumn{1}{c}{ Time } & \multicolumn{3}{c}{ UV Index } \\
\hline & A & B & Indoor \\
\hline 06.30 a.m & 1 & 0 & 0 \\
07.15 a.m & 2 & 1 & 0 \\
08.44 a.m & 4 & 3 & 0 \\
10.00 a.m & 5 & 5 & 0 \\
10.45 a.m & 6 & 6 & 0 \\
\hline
\end{tabular}

In testing ESP 32 as an IoT-based UV index reader we used two different locations, location A is at coordinates $-7.973731,112.579935$ and location $\mathrm{B}$ is at coordinates -7.9606839, 112.6191241. The data obtained from the tool is a UV index. The UV Index is a number without units to describe the level of exposure to ultraviolet radiation related to human health. By knowing the UV index we can monitor the level of ultraviolet rays that are beneficial and those that can provide harm. Each scale has a UV Index equivalent to $0.025 \mathrm{Wm} 2$ of ultraviolet radiation. The scale is obtained based on the spectral flux of UV radiation with a function corresponding to the photobiological effect on human skin, integrated between 250 and $400 \mathrm{~nm}$. The sensor calibration itself is based on the voltage issued by the sensor, the sensor used has been calibrated. From these two results, it is known that the two locations have an inequality in the UV index obtained. The difference in the UV Index obtained is caused by several factors, namely: the placement of the UV sensor which is blocked by leaves and the level of ozone damage in that place is different from the level of ozone damage in other areas. In the results of this experiment, the uv index of 0 does not mean there is no UV light, but the calculation of the tool rounds off v0 / 0.1. V0 numbers 0-0.99 are considered to be indexed UV 0 . With the right placement of tools and the right weather, we can study the level of ozone damage in an area by looking at the comparison of the UV index in one area with another area simultaneously. Based on the data obtained, the application of ESP 32 as a media for learning ozone damage in the form of IoT based UV index reader is running well.

\section{Conclusion}

WBased on the data we obtained from this research, we can conclude that the application of ESP 32 as a media for learning ozone damage in the form of IoT based UV index reader has worked well with some notes that internet connectivity is smooth and the placement of the UV sensor is not blocked by other objects or the UV sensor must be exposed to direct sunlight. 


\section{References}

[1] Alamsyah, Ardi, A., \& Faisal, M. N. (2015). Perancangan dan Penerapan Sistem Kontrol Peralatan Elektronik Jarak Jauh Berbasis Web (Design and Implementation of Web-Based Remote Electronic Equipment Control Systems). Journal of Mechanical, 6(2), 577-584.

[2] "Indeks Sinar Ultraviolet (UV) (Ultraviolet (UV) Light Index) | BMKG." [online]. Website: https://www.bmkg.go.id/cuaca/indeks-uv.bmkg, accessed date 26 June 2020

[3] Sulistyanto, M. T., Nugraha, D. A., dkk. (2015). 1. Implementasi IoT (Internet of Things) dalam pembelajaran di Universitas Kanjuruhan Malang (Implementation of IoT (Internet of Things) in learning at Kanjuruhan University Malang). SMARTICS Journal, 1(1), 20-23.

[4] T. B. Yulianto, A. J. Taufiq, and A. Suyadi, "Rancang Bangun Pengaturan Intensitas Sinar UV (Ultraviolet) Dengan Mikrokontroler PIC Untuk Tanaman (Design of UV (Ultraviolet) Intensity Settings with a PIC Microcontroller for Plants)” JRRE Jurnal Riset Rekayasa Elektro 1 (2019) 54-55

[5] T. Vivi, "Global warming," JKMA Jurnal Kesehatan Masyarakat (Journal of Public Health) Andalas 2 (2008) 159-160

[6] Zanella, A., \& Vangelista, L.. Internet of Things for Smart Cities. IEEE INTERNET OF THINGS JOURNAL, 1(1), 22-32. 